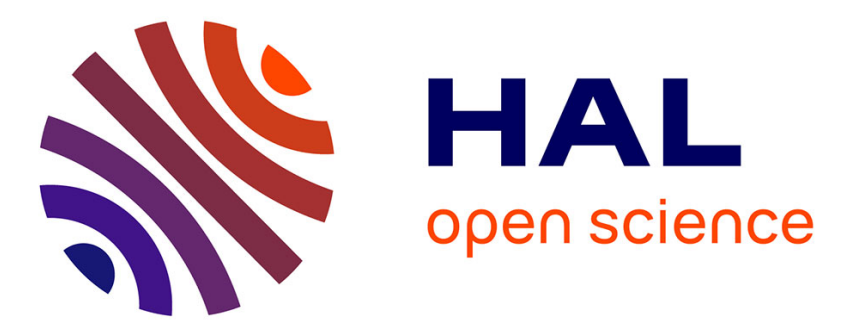

\title{
Roughening transition and formation of bicontinuous structures of immiscible solvents embedded in surfactant diblock copolymers
}

\author{
A. Robledo, C. Varea, E. Martina
}

\section{- To cite this version:}

A. Robledo, C. Varea, E. Martina. Roughening transition and formation of bicontinuous structures of immiscible solvents embedded in surfactant diblock copolymers. Journal de Physique Lettres, 1985, 46 (20), pp.967-972. 10.1051/jphyslet:019850046020096700 . jpa-00232925

HAL Id: jpa-00232925

https://hal.science/jpa-00232925

Submitted on 1 Jan 1985

HAL is a multi-disciplinary open access archive for the deposit and dissemination of scientific research documents, whether they are published or not. The documents may come from teaching and research institutions in France or abroad, or from public or private research centers.
L'archive ouverte pluridisciplinaire HAL, est destinée au dépôt et à la diffusion de documents scientifiques de niveau recherche, publiés ou non, émanant des établissements d'enseignement et de recherche français ou étrangers, des laboratoires publics ou privés. 
Classification

Physics Abstracts

$64.60-68.10-82.70$

\title{
Roughening transition and formation of bicontinuous structures of immiscible solvents embedded in surfactant diblock copolymers
}

\author{
A. Robledo (*), C. Varea \\ División de Estudios de Posgrado, Facultad de Química, \\ Universidad Nacional Autonóma de México, 04510 México, D.F. \\ and E. Martina \\ Instituto de Física, Universidad Nacional Autónoma de México, Apartado Postal 20-364, \\ 01000 México, D. F.
}

(Reçu le 26 juin 1985, accepté sous forme définitive le 30 août 1985)

\begin{abstract}
Résumé. - On examine la transition de rugosité de l'interphase dans un mélange modèle constitué de copolymères diblocs amphiphiles de deux solvants non miscibles et d'un cosurfactant monomérique. L'équivalence entre ce modèle et celui du solide-sur-solide implique l'existence d'une composition critique $X_{\mathrm{AB}}^{\mathrm{R}}$ du cosurfactant pour que la transformation d'une phase de micro-émulsion lamellaire ait lieu dans une structure désordonnée bicontinue. A une composition plus élevée $1-X_{\mathrm{AB}}^{\mathrm{R}}$, une autre transition produit de nouveau une phase ordonnée.
\end{abstract}

\begin{abstract}
The roughening transition of the interface in a model mixture of amphiphilic diblock copolymers, two immiscible solvents and a monomeric cosurfactant is examined. The equivalence of this model with the solid-on-solid Ising magnet implies the existence of a critical cosurfactant composition $X_{\mathrm{AB}}^{\mathrm{R}}$ for the transformation of a lamellar microemulsion phase into a bicontinuous disordered structure. At a higher composition $1-X_{\mathrm{AB}}^{\mathrm{R}}$ another transition of the same nature produces again an ordered phase.
\end{abstract}

In this Letter we address the problem of the formation of bicontinuous microemulsion phases - where the regions of each immiscible solvent are connected over macroscopic distances in multicomponent fluid mixtures where amphiphilic species are present. We show that the Kosterlitz-Thouless (KT) [1, 2] theory of phase transitions in two-dimensional systems provides a mechanism, at least within a particular model system where one amphiphile is polymeric, of how a disordered bicontinuous phase can be produced from phases with multilamellar arrangements.

Our contention here is that the nature of a bicontinuous microemulsion phase is that of a set of delocalized (or rough) [3] interfaces, so that a multisurface arrangement, obtained when the surfactant concentration is many times that needed to saturate one interface, is necessarily

(*) J. S. Guggenheim fellow. 
disordered; i.e. the surfactant(s) film has a random nonperiodic structure throughout the sample volume. The roughening transition associated with the disappearance of a sharply defined orientation of an interface constitutes a mechanism by means of which ordered phases of immiscible solvents separated by localized interfaces transform into disordered structures. The appearance of disorder at a critical cosurfactant composition $X_{A B}^{R}$ is signalled by the vanishing of the free-energy cost associated to provide curvature to a planar interface. We find, for $X_{\mathrm{AB}}>X_{\mathrm{AB}}^{\mathrm{R}}$, that the positional correlation function between two pieces of the same wrinkled (or rough) interface, separated by a distance $r$, decays algebraically like the spin correlations in the low temperature phase of the $X Y$ magnet. As a consequence, a positional persistence length $\xi_{\mathrm{R}}$, analogous to that introduced by de Gennes and Taupin [4] for orientations of the surfactant film, acquires a well defined finite value. Thus, the rough interface is approximately flat only at scales smaller than $\xi_{R}$ and consecutive " pieces " of area $\xi_{R}^{2}$ have independent positions. We find, as in reference [4], that $\xi_{\mathrm{R}}$ depends exponentially on the parameter that measures interfacial rigidity.

Our model mixture is closely related to that discussed by Cantor [5] in his study of the interfacial properties of non-ionic diblock copolymer amphiphiles. These kind of surfactants consist of two different polymeric chains joined together, each of which strongly prefers one solvent. A water/oil interface saturated with such polymer chains has the hydrophilic blocks embedded, or swollen, by water and, similarly, the hydrophobic portions by oil. Cantor considered the formation of an ordered " middle " phase in a three component system. The particular arrangement studied was a multilamellar phase between two solvent phases composed of alternating layers of the two solvents stabilized at their interfaces by the chains. Here we analyse the possibility of transforming, isothermally, such ordered structure into a disordered bicontinuous one through the addition of a fourth component, a monomeric cosurfactant that increases the flexibility [4] of the layers embedded with solvent. We assume that the cosurfactant species is always preferentially located at solvent-solvent interfacial regions.

But also, our model mixture is a variant of the Wheeler-Widom (WW) lattice mixture of bifunctional molecules [6]. The WW model abstracts some of the essential features of a ternary system composed of water, oil and surfactant. The interactions amongst molecules are simplified to a maximum that only retains (through infinite repulsions or no attractions) the basic character of each constituent. In its simplest version [6] the model is isomorphous to the Ising model in the same lattice, and displays as corresponding states both ferromagnetic and antiferromagnetic behaviour. The binodal curve and plait point of the mixture are images of the spontaneous magnetization and Curie point of the ferromagnet, respectively. At high concentrations of surfactant antiferromagnetic-like ordering appears. The model is composed of bifunctional molecules $\mathrm{AA}(\longrightarrow), \mathrm{BB}\left(\mathrm{O}_{-}\right)$and $\mathrm{AB}(-\mathrm{O})$, and it is constructed by : (i) confining them to the bonds of a regular (in our case, three-dimensional) lattice; (ii) letting only A atoms or only $B$ atoms meet at any lattice site ( $A$ ends of one molecule and $B$ ends of another exclude each other); and (iii) filling every bond of the lattice with one, and only one, molecule. Since for all allowed configurations every site can be identified as either an A or a B site, the equivalence with the Ising model follows. The complete transcription to fluid mixture language, the model's phase diagram and the mean-field description of the interface between coexisting phases is given in reference [6]. There, the consideration of micellar structures in different regions of the phase diagram, the behaviour of the liquid-liquid interfacial tension and other matters pertaining to the WW model are discussed. Here we shall focus attention on other issues. Because every bond in the lattice is filled by one molecule the chemical potentials $\mu_{\mathrm{AA}}, \mu_{\mathrm{B} B}$ and $\mu_{\mathrm{AB}}$ of the three species are all infinite, but the difference of any pair is in general finite. In particular, when the mixture and the magnet are set at the same temperature, the chemical potential differences are related to the Ising coupling $J$ by

$$
\tilde{\mu} \equiv \mu_{\mathrm{AB}}-\frac{1}{2}\left(\mu_{\mathrm{AA}}+\mu_{\mathrm{BB}}\right)=-2 J .
$$


We call $\tilde{\mu}$ the (relevant) surfactant chemical potential. Since the potential energy of every allowable configuration always vanishes, the mixture's temperature does not play a relevant role. We shall be interested only in the behaviour corresponding to the anisotropic limit of the Ising model in which the vertical coupling $J_{z}$ approaches infinity, giving the solid-on-solid (SOS) [3, 7] version of the coexisting interphase. In this limit no new vertical " broken bonds » can occur, so bulk excitations and overhanging configurations are suppressed and the critical Ising $T_{\mathrm{c}}$ tends to infinity. This particular limit is obtained from the WW model if we consider a diblock copolymer to be composed of two large chains of $\mathrm{AA}$ and $\mathrm{BB}$ monomers joined by an $\mathrm{AB}$ bond. If the polymers saturate an interface normal to the $z$-axis the divergence of $J_{z}$ ensures that the chains are permanent entities occupying all of the vertical bonds in the lattice. They constitute the SOS columns with their heights given by the positions of the $A B$ junctions. The embedded solvents AA and BB occupy only horizontal bonds in the lattice and connect sites occupied by polymer chains of the same kind, whereas the monomeric cosurfactant $\mathrm{AB}$ joins portions of neighbouring chains that are of different kind. When there is no cosurfactant all SOS columns for a strictly planar interface and additions of cosurfactant produce shifts in column heights and introduce disorder at the interface. (See Fig. 1a.) We have considered the copolymer chains to be rigid and to be in a close-packed state. They are therefore unable to reproduce the polymer fluctuations in Cantor's middle-phase model. Here we only take advantage of the difference in size between surfactant and cosurfactant molecules to study interfacial events in this limiting case.

The behaviour of the mixture is easily understood when the correspondence of states with the magnet is established at fixed temperature. Then, from equation (1) we observe that addition of cosurfactant $\mathrm{AB}$ to a mixture with a given $\mathrm{AA}$ to $\mathrm{BB}$ ratio (say $\mu_{\mathrm{AA}}-\mu_{\mathrm{BB}}$ fixed) implies a reduction of the Ising coupling constant at the same temperature. $J_{x}=J_{y}=J \rightarrow \infty$ at the base line $X_{\mathrm{AB}}=0, J$ changes sign at a $X_{\mathrm{AB}}=\frac{1}{2}$ and $\tilde{\mu}=0$ (when the spins are uncoupled and the
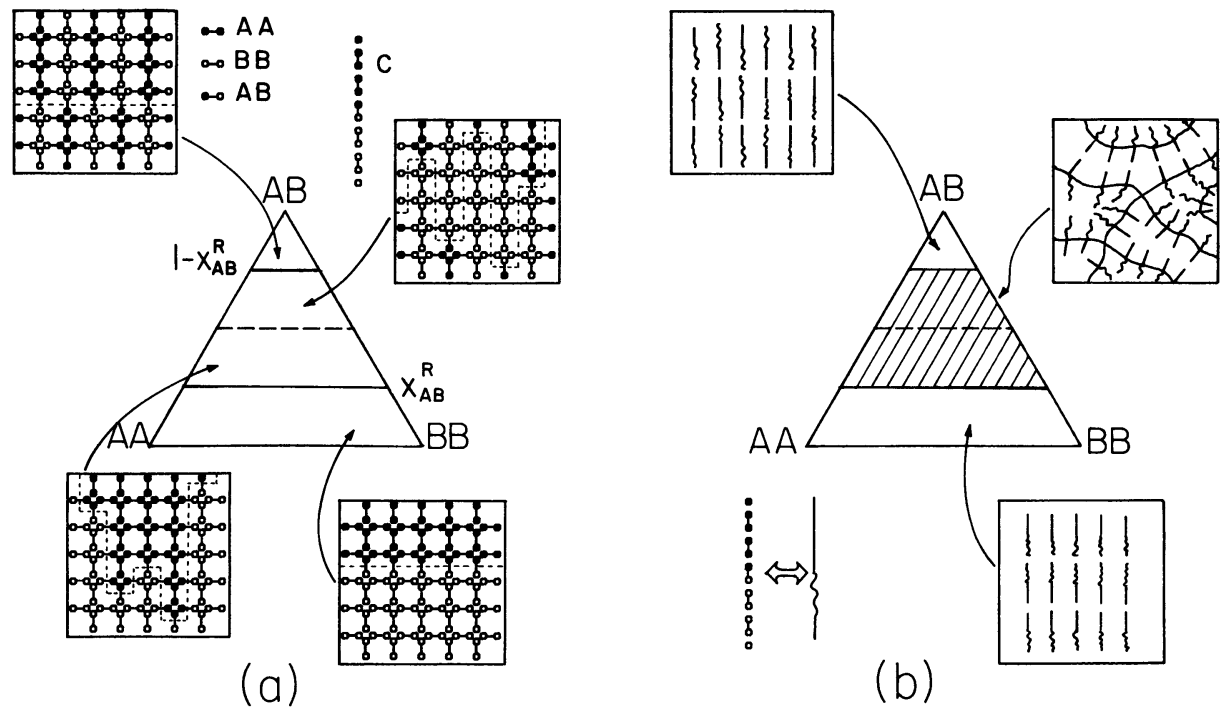

Fig. 1. - (a) Composition triangle for the horizontal bonds in a single SOS model mixture. The different microscopic structures in each region of composition are also shown. $\mathrm{C}$ is the copolymer, $\mathrm{AB}$ the cosurfactant and AA and BB the immiscible solvents. (b) Phase diagram for the multisurface microemulsion model represented by a sequence of stacked SOS systems. The shaded region gives the composition range for the disordered microemulsion while the unshaded regions give those of the lamellar-ordered phases. The microscopic structures which correspond to each region are shown. 
SOS columns behave independently), and finally $J \rightarrow-\infty$ for the binary surfactant-cosurfactant system at $X_{\mathrm{AB}}=1$. Pure AA and BB phases coexist separated by a monolayer of diblock copolymer until $\tilde{\mu}=0$ when the turnover to antiferromagnetic behaviour takes place. Thereafter, as $X_{\mathrm{AB}}$ is incremented, the repulsion between SOS columns favours ordered columnar structures that become a regular arrangement of alternating up and down copolymer molecules at $X_{\mathrm{AB}}=1$ (see Fig. 1a). Following Cantor [5], if the relative concentration of copolymer chains is many times that needed to saturate one interface the system may form many flat slices of the solvents between the two bulk solvent phases, i.e. a middle-phase lamellar microemulsion. When the copolymer concentration is sufficiently high the multilayer phase exhausts either or both of these phases. Cantor concluded [5], under the consideration of long-range van der Waals forces and steric repulsions between the chains, that the multilayer phase corresponds to alternating layers (of width $2 M$ ) of the copolymer chains (each of length $2 M$ ). In our model mixture, the properties of the multilayer phase are given by those of a sequence of stacked SOS systems where the columns between two neighbouring SOS systems show blocks of one kind followed by blocks of the opposite kind in the $z$-direction (see Fig. 1b). This set of SOS systems can be seen to form an enlarged SOS model where each column now, possibly of infinite length, is a chain of copolymer molecules.

It is clear that when a roughening transition occurs at the interface of the SOS Ising model its exact image appears at the corresponding interface of the SOS version of the WW model. Roughening can be observed in the magnet at fixed $T$ if $J$ is varied and through equation (1) the corresponding chemical potential, $\tilde{\mu}_{\mathrm{R}}$, or roughening composition $X_{\mathrm{AB}}^{\mathrm{R}}$, is obtained. The existence of a roughening transition in the SOS model defined in the body centred cubic lattice has been proved rigorously $\left(J_{\mathrm{R}}=k_{\mathrm{B}} T \ln \sqrt{2}, k_{\mathrm{B}}\right.$ Boltzmann's constant) [8], and in computer experiments on other SOS models the existence of a roughening transition has been shown fairly convincingly [9]. The connection between roughening in SOS models and the KT theory of 2-d transitions has been discussed in detail [3]. The KT theory proposes a novel mechanism for a phase transition in 2-d systems that lack conventional long-range order but involve a change in their topological order. A host of two dimensional problems appear to exhibit this behaviour and to be closely related to each other [10-12]. The renormalized Kosterlitz-Thouless coupling $[1,10], K_{X Y}^{\text {eff }}$ of the dual $X Y$ model induces a universal jump (in appropriate units) in the mixture's $\tilde{\mu}$. We recall that $K_{X Y}^{\text {eff }}$ vanishes for $K_{X Y}<K_{X Y}^{\mathrm{c}}$ and is equal to $2 / \pi$ at $K_{X Y}^{\mathrm{c}}$, where $K_{X Y}=$ $J^{\prime} / k_{\mathrm{B}} T$ is the dimensionless coupling in dual variables $\left(J^{\prime}=1 / J\right.$ and $\left.T^{\prime}=1 / T\right)$. The mixture's image of $K_{X Y}^{\text {eff }}$ is the effective cosurfactant chemical potential $\tilde{\mu}_{\text {eff }}=-k_{\mathrm{B}} T / 2 K_{X Y}^{\text {eff }},-\tilde{\mu}_{\text {eff }}$ is infinite when $\tilde{\mu}<\tilde{\mu}_{\mathrm{R}}$ and jumps to $\pi k_{\mathrm{B}} T / 4$ at $\tilde{\mu}=\tilde{\mu}_{\mathrm{R}}$. Another manifestation of the KT theory is the vanishing of the free energy required to give curvature $\kappa$ to a flat interface, $f_{\kappa}$. Since an interface with macroscopic curvature $\kappa$ can be formed from a flat one by a sequence of single steps, the vanishing of the step free-energy [8] $\left(\operatorname{as} \exp \left(-\alpha\left|\left(\tilde{\mu}_{R}-\tilde{\mu}\right) / \tilde{\mu}\right|^{-1 / 2}\right)\right.$ is transmitted to $f_{\kappa}$.

An important correlation function in the study of the roughening transition in SOS models is

$$
g(r)=\int \mathrm{d} z z^{2} P(z ; r)=\left[(h(0)-h(r))^{2}\right],
$$

where $P(z ; r)$ is the probability that the height difference of two columns, a distance $r$ apart, is equal to $z$. The interfacial width $L$ is obtained through $L^{2}=\lim _{r \rightarrow \infty} g(r)$. The behaviour of $g(r)$ is unbounded when $X_{\mathrm{AB}}>X_{\mathrm{AB}}^{\mathrm{R}}, g(r) \sim\left(4 k_{\mathrm{B}} T a^{2} / \pi\left|\tilde{\mu}_{\mathrm{eff}}\right|\right) \times \ln (r / a)$, and $L$ is always infinite for an infinite system ( $a$ is the lattice spacing). In contrast, for $X_{\mathrm{AB}}<X_{\mathrm{AB}}^{\mathrm{R}}, g(r)$ behaves as $g(r) \sim 2 / \pi^{2} \ln \xi_{\mathrm{L}} / a$ when $r / \xi_{\mathrm{L}} \rightarrow \infty$. The interfacial width correlation length $\xi_{\mathrm{L}}$ diverges as $\exp \left(\alpha\left|\left(\tilde{\mu}_{\mathbf{R}}-\tilde{\mu}\right) / \tilde{\mu}\right|^{-1 / 2}\right)$ near $X_{\mathbf{A B}}^{\mathbf{R}}$. It is convenient to express $P(z ; r)$ using the characteristic 
function

$$
E(k ; r)=\frac{1}{2 \pi} \int \mathrm{d} z \mathrm{e}^{-i k z} P(z ; r)=\left[\mathrm{e}^{i k(h(0)-h(r))}\right] .
$$

This function, to first order in a cumulant expansion, is given by [10] $E(k ; r) \sim \exp \left(-k^{2} / 2 g(r)\right)$. The maximum permissible column height difference in our model interface is $M$, and therefore $E\left(k_{\min }=2 \pi / M ; r\right)=\exp \left(-\frac{1}{2}(2 \pi a / M)^{2} g(r)\right)$ collects all contributions to the column correlations up to this height difference. Now, in analogy with reference [4], we can define a positional persistence length $\xi_{\mathbb{R}}$, according to which the interface appears flat at short scales $\left(r<\xi_{\mathbb{R}}\right)$ and rough at large scales $\left(r>\xi_{R}\right)$. Use of the asymptotic form of $g(r)$ in $E(2 \pi / M ; r)$ yields

$$
E(2 \pi / M ; r)=\left|\frac{a}{r}\right| k_{\mathrm{B}} T / 2 \pi R .
$$

Where $R=\left|\tilde{\mu}_{\text {eff }}\right|(M / 4 \pi a)^{2}$, and by choosing $\xi_{\mathrm{R}}$ such that $E(2 \pi / M ; r)=1 / \mathrm{e}$ it follows that $\xi_{\mathrm{R}}=a \exp \left(2 \pi R / k_{\mathrm{B}} T\right)$. The quantity $R$ plays the role of Helfrich's rigidity constant associated to an interface saturated by surfactant [4]. Roughening transitions have been calculated, and rigorous proofs provided [13], only in relation to lattice-space models. The interfacial transition we describe on a lattice corresponds to a multicomponent layered liquid crystal (a smectic-B type phase) and not to an isotropic fluid for which continuum space is more appropriate. The $\mathrm{KT}$ mechanism of phase transitions has been associated to phenomena occurring in real (thermotropic) liquid crystal films [14].

If the system of size $A^{3}$ contains only one rough interface, this could wander only over a vertical extent $\sim \ln \Lambda$. However, the roughening of the interfaces that constitute a multilamellar phase, also described, as pointed out above, by an enlarged SOS system, produces a disordered structure since they acquire a random delocalized state in exactly the same way as a single SOS system representing only one monolayer of copolymer (in the $M \rightarrow \infty$ limit). We have assumed, in accordance to Cantor findings [5], that the solvents cannot accumulate and separate successive layers of copolymer. We think of the multilamellar phase as represented by sequences of copolymer molecules permanently touching at their ends and occupying all vertical lattice bonds. This requires the divergence of the $J_{z}$ that represents chain-chain contacts. As a result of roughening, regions embedded by each solvent may become multiply connected and generate a bicontinuous structure (see Fig. 1b) [4]. In the rough phase interfacial surfaces wander many times the block copolymer length $M$ along the $z$-direction and the majority of the allowed configurations contain same-solvent paths that visit regions that were in different layers in the ordered phase. There are however other, but combinatorially fewer, configurations that retain the individual character of the layers. An explicit proof of this crucial point is not provided in this occasion and therefore this remains a plausibility argument. Bicontinuous structures can only exist for a range of values of cosurfactant composition since another roughening transition, occurring for large $X_{\mathrm{AB}}$, transforms the system back into an ordered phase (that differs in geometry from the low $X_{\mathrm{AB}}$ multilamellar phase). This other transition corresponds to the roughening of the interface of the antiphase boundary of the antiferromagnetic SOS model (see Fig. 1b). Since the two kinds of SOS models can be obtained from each other by only changing the sign of $J$, the latter transition occurs at $1-X_{\mathrm{AB}}^{\mathrm{R}}$. In all other respects the two transitions are identical since the relevant properties are determined by $\left|\tilde{\mu}_{\text {eff }}\right|$. The physical meaning of $\left|\tilde{\mu}_{\text {eff }}\right|$ is provided by its role in defining the positional persistence length $\xi_{R}$. It is therefore a measure of the rigidity of the interface and, as we have seen, it undergoes a universal jump, like its counterparts in other KT problems [14], at the transitions. It is infinite for the layered phases and finite for the disordered one. The most noticeable difference between ordered and disordered microemulsion phases in real systems is related to their distinct strain and flow properties. 


\section{Acknowledgments.}

Two of us (CV and AR) acknowledge financial support by Consejo Nacional de Ciencia y Tecnología de México.

\section{References}

[1] Kosterlitz, J. M. and Thouless, K. J., J. Phys. C 6 (1973) 1181 ; Kosterlitz, J. M., J. Phys. C 7 (1974) 1046.

[2] Kosterlitz, J. M. and Thouless, K. J., Prog. Low Temp. Phys. B 7 (1978) 371 ; BARber, M. N., Phys. Rep. 59 (1980) 375.

[3] Chui, S. T. and Weeks, J. D., Phys. Rev. B 14 (1976) 4978;

Knops, H. J. F., Phys. Rev. Lett. 39 (1977) 766.

See also Ohta, T. and Kawasaki, K., Prog. Theor. Phys. 60 (1978) 365.

[4] De Gennes, P.. G. and Taupin, C., J. Phys. Chem. 86 (1982) 2294.

[5] CANTOR, R., Macromolecules 14 (1981) 1186.

[6] Wheeler, J. C. and Widom, B., J. Am. Chem. Soc. 90 (1968) 3064 ; Widom, B. J., Phys. Chem. 88 (1984) 6508.

[7] SWEndSEn, R. H., Phys. Rev. B 15 (1977) 689.

[8] Van BeiJeren, H., Phys. Rev. Lett. 38 (1977) 993.

[9] Leamy, H. J. and Jackson, K. A., J. Appl. Phys. 42 (1971) 2121 ; Gilmer, G. H. and Bennema, P., J. Appl. Phys. 43 (1972) 1347. LeAmy, H. J. and Gilmer, G. H., J. Cryst. Growth 24/25 (1974) 499.

[10] José, J. V., Kadanoff, L. P., KirkPatrick, S. and Nelson, D. R., Phys. Rev. B 16 (1977) 1217 ; Ohta, T. and Kawasaki, K., Prog. Theor. Phys. 60 (1978) 365.

[11] Nelson, D. R. and Kosterlitz, J. M., Phys. Rev. Lett. 39 (1977) 1201 ; Nelson, D. R. and Toner, J., Phys. Rev. 1324 (1981) 363.

[12] Müller-KrumbhaAR, H., Developments in the Theory of the Roughening Transition in Crystal Growth and Materials. Eds. E. Kaldis and H. Y. Scheel (Amsterdam) 1977.

[13] Fröhlich, J. and SPEnCER, T., Commun. Math. Phys. 81 (1981) 527.

[14] Pindak, R. and Moncton, D., Phys. Today (May issue) 57 (1982). 